\title{
Approximation of Conformal Mappings via Bieberbach Polynomials inside Regions with Cusps
}

\author{
Cem KOŞAR* \\ Gaziantep University, Nizip Education Faculty, Mathematics and Science Education Department, Gaziantep \\ (ORCID: 0000-0003-4949-4956)
}

\begin{abstract}
In this work, some estimates for the Bieberbach polynomial approximation of the conformal mapping of inside the finite simple connected region with simultaneously cusps onto the disc in the complex plane are obtained. Moreover, the speed of the approximation depends on the boundary property of the region.

Keywords: Conformal mappings, quasiconformal curve, Bieberbach polynomials

\section{Sıfır Açılı Bölgelerin içinde Bieberbach Polinomları ile Konform Dönüşümlerin Yaklaşımı}

$\ddot{\mathbf{O} z}$

Bu çalışmada, kompleks düzlemin; sonlu, basit bağlantılı ve aynı zamanda hem iç sıfır açı hem de dıș sıfır açıya sahip bölgelerin içerisinde konform dönüşümlere Bieberbach polinomlarıyla yaklaşımı için bazı hesaplamalar elde edimiştir. Ayrıca yaklaşımın hızı bölgenin sınır özelliğine bağlıdır.

Anahtar kelimeler: Konform dönüşümler, yarıkonform eğri, Bieberbach Polinomları.

\section{Introduction}

\subsection{Statement of the Problem}

For convenience, let us denote by $\mathrm{G}$ that is a Jordan region in the $z$-plane, which is bounded by rectifiable Jordan curve $\Gamma:=\partial G$ and let $z_{0}$ belongs to $\mathrm{G}$. We know that there is a unique conformal function $w=\varphi\left(z ; z_{0}\right)$ acting from $\mathrm{G}$ to the disk $D\left(0 ; r_{0}\right)$ normalized by $\varphi\left(z_{0} ; z_{0}\right)=0, \varphi^{\prime}\left(z_{0} ; z_{0}\right)=1$. Let us also represent by $\wp_{n}$ the class of all polynomials $p_{n}$ with degree not exceeding $\mathrm{n}$ and satisfies the conditions $p_{n}\left(z_{0} ; z_{0}\right)=0, p_{n}^{\prime}\left(z_{0} ; z_{0}\right)=1$. The Bieberbach polynomials $\pi_{n}\left(z ; z_{0}\right)$ are solution of the extremal problem in the Bergman space. Furthermore, it is clear that Bieberbach polynomial has also a property for the minimization of the norm $\left\|\varphi^{\prime}-p_{n}^{\prime}\right\|_{L_{2}(G)}$ in the class $\wp_{n}$.

Let $z_{0} \in B$ an arbitrary closed disk subset of $\mathrm{G}$. It is obvious that if $G$ is a Caratheodory region, then $\left\|\varphi^{\prime}-p_{n}^{\prime}\right\|_{L_{2}(G)}$ tends to zero as $n \rightarrow \infty$, and so the Bieberbach sequence $\left\{\pi_{n}\left(z ; z_{0}\right)\right\}_{n=1}^{\infty}$ goes uniformly convergence to $\varphi\left(z ; z_{0}\right)$ on compact subset of $G$. Therefore, all $z, z_{0} \in B$

$$
\omega_{n}(B):=\sup _{z, z_{0} \in B}\left|\varphi\left(z ; z_{0}\right)-\pi_{n}\left(z ; z_{0}\right)\right| \rightarrow 0
$$

*Sorumlu yazar: ckosar77@gmail.com

Geliş Tarihi: 01.11.2019, Kabul Tarihi: 25.11.2019 
Keldych [15] was the first to investigate the uniform convergence of the Bieberbach polynomials in the region $\bar{G}$. Furthermore, the improvements in such the extremal problem has been studied in $[2,4,5,9-14,18,20-22]$ and the others. In the approximation theory, the rate of approximation of a given function in the region $G$ is better than the rate of the approximation in $\bar{G}$. For which regions are this property valid with respect to the approximations by Bieberbach polynomials. Firstly, Suetin [21] studied this problem for regions $G$ with $\Gamma \in C(p+1, \alpha), p \geq 0,0<\alpha<1$ and obtained the following estimation for (1.1):

$$
\omega_{n}(B) \leq c[\operatorname{dist}(B, \Gamma)]^{-2(p+3)} n^{-2(p+\alpha)} .
$$

Then, this research was investigated by $[6,16]$ in various regions of the complex plane. In this work, we propose to study the assessment

$$
\omega_{n}(B) \leq c \delta^{-q}(B) \eta_{n}, \quad \delta(B)=\operatorname{dist}(B, \Gamma),
$$

where $\mathrm{c}$ is a constant, $q>0$ and $\eta_{n} \rightarrow 0$, as $n \rightarrow \infty$ in a different region. $[3,7]$.

The purpose of this study is to apply the problem (1.3) to the class of $\widetilde{P Q}(K, \alpha, \beta)$ defined in

\section{Main Theorems}

The next theorems are our main outcomes of this study:

Theorem 2.1. Let $G \in \widetilde{P Q}(K, \alpha, \beta)$ for some $K>1,0<\alpha<1 / 3$ and $0<\beta<(1-3 \alpha) / 2 \alpha$. Then there exists constant $c=c(\Gamma)$ for any $n \geq 2$

$$
\omega_{n}(B) \leq c \delta^{-3}(B)\left(\frac{1}{\ln n}\right)^{\frac{1-3 \alpha}{2 \alpha}-\beta} .
$$

Theorem 2.2. Let $G \in \widetilde{P Q}(K, \alpha, 0)$ for some $K>1$ and $0<\alpha<1 / 2$. Then there exists constant $c=c(\Gamma)$ for any $n \geq 2$

$$
\omega_{n}(B) \leq c \delta^{-3}(B)\left(\frac{1}{\ln n}\right)^{\frac{1-2 \alpha}{2 \alpha}}
$$

Theorem 2.3. Let $G \in \widetilde{P Q}(K, 0, \beta)$ for some $K>1$. Then there exists constant $c=c(\Gamma)$ for any $n \geq 2$

$$
\omega_{n}(B) \leq c \delta^{-3}(B)(\ln n)^{\frac{5 \beta}{2}+1}\left(\frac{1}{n}\right)^{\frac{1}{2 K^{2}}} .
$$

\section{Some Auxiliary Facts}

In this section, we need some auxiliary facts to obtain the main results. Throughout this study $c, c_{1}, c_{2}, c_{3}, \ldots$ denote positively fixed numbers and $\varepsilon_{1}, \varepsilon_{2}, \varepsilon_{3}, \ldots$ are small enough positively fixed. Moreover, positive constants are not necessarily the same at different places. The notation $a \leqslant b$ means that $a \leq c_{1} b$ for $c_{1}$, which is independent of $a$ and $b$. The symbol $a \approx b$ indicates that $c_{3} b \leq a \leq c_{4} b$, where $c_{3}, c_{4}$ does not depend on $a$ and $b$. 
For an arbitrary $z_{0} \in B$, let $w=\varphi_{0}\left(z ; z_{0}\right)$ be conformal mapping acting from $G$ to the unit disc with the conditions $\varphi_{0}\left(z_{0} ; z_{0}\right)=0, \varphi_{0}^{\prime}\left(z_{0} ; z_{0}\right)>0$. Whenever we write $w=\varphi_{0}(z)$, it will be understood that $w=\varphi_{0}\left(z ; z_{0}\right)$ for a constant point $z_{0}$.

Let $w=\Phi(z)$ be a conformal function acting from $\Omega:=\operatorname{ext} \bar{G}$ to $\Delta:=\{w:|w|>1\}$, with the conditions $\Phi(\infty)=\infty, \lim _{z \rightarrow \infty} \Phi(z) / z>0$. We first need to convene on some notations for $\delta>0$,

$$
\begin{gathered}
\Gamma_{\delta}:=\left\{z:\left|\varphi_{0}\left(z ; z_{0}\right)\right|=\delta, \quad \delta<1\right\}, \quad \Gamma_{\delta}:=\{z:|\Phi(\mathrm{z})|=\delta, \quad \delta>1\} \\
\Gamma_{1}=\Gamma, G_{\delta}:=\operatorname{int}\left(\Gamma_{\delta}\right), \Omega_{\delta}:=\operatorname{ext}\left(\Gamma_{\delta}\right)
\end{gathered}
$$

We know that if $\Gamma$ be a $K$-quasiconformal curve, then there is a $\alpha^{*}($.$) quasiconformal$ reflection. By using the events in [8, p.76], it could be found a $C(K)$-quasiconformal reflection $\alpha^{*}($. such that the next conditions

$$
\begin{gathered}
\left|\xi-\alpha^{*}(z)\right| \approx|\xi-z|, \quad \xi \in \Gamma ;\left|\alpha_{\bar{z}}^{*}\right| \approx\left|\alpha_{z}^{*}\right| \approx 1, \quad \varepsilon<|z|<\varepsilon^{-1}, \\
\left|\alpha_{\bar{z}}^{*}\right| \approx\left|\alpha_{z}^{*}\right|^{2},|z|<\varepsilon ;\left|\alpha_{\bar{z}}^{*}\right| \approx z^{-2},|z|>\varepsilon^{-1}, \\
\left|\alpha^{*}(z)-\xi\right| \approx|z-\xi|, \xi \in \Gamma ; J_{\alpha^{*}}:=\left|\alpha_{z}^{*}\right|^{2}-\left|\alpha_{\bar{z}}^{*}\right|^{2} \approx 1 .
\end{gathered}
$$

are held in some neighborhood of $\Gamma[1]$.

From now on, we will choose for simplicity the cusps of the class $\widetilde{P Q}(K, \alpha, \beta)$ as in [7]. Thus, it could be obtained from [7, Lemma 2.1]

$$
\begin{gathered}
\mathrm{d}(\mathrm{z}, \Gamma) \preccurlyeq\left(\left|\varphi_{0}\left(\mathrm{z} ; \mathrm{z}_{0}\right)\right|-1\right)^{\mathrm{K}^{-2}} ; \\
|\mathrm{z}-1| \preccurlyeq\left|\varphi_{0}\left(\mathrm{z} ; \mathrm{z}_{0}\right)-\varphi_{0}\left(1 ; \mathrm{z}_{0}\right)\right|^{\mathrm{K}^{-2}},|\mathrm{z}+1|>\varepsilon_{1} \\
\mathrm{~d}(\mathrm{z}, \Gamma) \preccurlyeq(|\Phi(\mathrm{z})|-1)^{\mathrm{K}^{-2}} ; \\
|\mathrm{z}+1| \preccurlyeq|\Phi(\mathrm{z})-\Phi(-1)|^{\mathrm{K}^{-2}},|\mathrm{z}-1|>\varepsilon_{2} .
\end{gathered}
$$

Lemma 3.1. [7] Let $G \in \widetilde{P Q}(K, \alpha, \beta)$ for some $K>1,0<\alpha<1, \beta \geq 0$ and $v(t)=\sqrt{t}$. Then

$$
\left\|F_{\gamma}^{\prime}\right\|_{L_{2}(G)} \leqslant l^{\frac{1-\alpha}{2}}
$$

Lemma 3.2. [7] Let $G \in \widetilde{P Q}(K, 0, \beta)$ for some $K>1, \quad \beta>0$ and $v(t)=t(-\ln t)^{-\beta}$. Then

$$
\left\|F_{\gamma}^{\prime}\right\|_{L_{2}(G)} \preccurlyeq l^{\frac{1}{2}}|\ln l|^{\beta}
$$

Lemma 3.3. [7] Let $G \in \widetilde{P Q}(K, \alpha, \beta)$ for some $K>1, \alpha \geq 0, \beta>0$. Then for all $\zeta \in G, z \in \Gamma$ the following holds:

$$
\left|\varphi_{0}\left(z ; z_{0}\right)-\varphi_{0}\left(\zeta ; z_{0}\right)\right| \preccurlyeq \delta(B)^{-\frac{1}{2}}\left\{\begin{array}{cc}
|z-\zeta|^{\frac{1}{2}}(-\ln |z-\zeta|)^{\frac{\beta}{2}} & ; \beta>0 \\
|z-\zeta|^{\frac{1}{2}} & ; \beta=0
\end{array}\right.
$$

\section{Approximation by Bieberbach Polynomials in the Bergman Space:}

Let us assume that a region $G \in \widetilde{P Q}(K, \alpha, \beta), K>1, \alpha \geq 0, \beta>0$ is got as in [7]. Each $\Gamma^{j}, j=1,2$ is a $K_{j}$ - quasiconformal arc and $\alpha_{j}^{*}($.$) is a quasiconformal reflection across \Gamma^{j}$. We also establish: 


$$
\begin{gathered}
\gamma_{1}^{1}:=\left\{z=x+i y: y=\frac{2 c_{1}+c_{2}}{3}(x-1)^{1+\alpha}\right\} ; \\
\gamma_{1}^{2}:=\left\{z=x+i y: y=\frac{c_{1}+2 c_{2}}{3}(x-1)^{1+\alpha}\right\} ; \\
\gamma_{2}^{1}:=\alpha_{1}^{*}\left\{z=x+i y: y=\frac{2 c_{3}+c_{4}}{3}(x+1)(-\ln (x+1))^{-\beta}\right\} ; \\
\gamma_{2}^{2}:=\alpha_{2}^{*}\left\{z=x+i y: y=\frac{c_{3}+2 c_{4}}{3}(x+1)(-\ln (x+1))^{-\beta}\right\} .
\end{gathered}
$$

Let $R=1+c n^{\varepsilon-1}$. It is also chosen points $z_{j}^{i},(i, j=\overline{1,2})$ such that these points are combined $\Gamma_{R}$ with $\gamma_{j}^{i}$ are in the first such points in $\Gamma_{R}^{1}:=\left\{z \in \Gamma_{R}: \operatorname{Im} z \geq 0\right\}$ or $\Gamma_{R}^{2}:=\Gamma_{R} \backslash \Gamma_{R}^{1}$. These points divide $\Gamma_{R}$ into four parts: $\Gamma_{R}^{1}:=\Gamma_{R}^{1}\left(z_{1}^{1}, z_{2}^{1}\right)$ with the endpoints $z_{1}^{1}$ and $z_{2}^{1}, \Gamma_{R}^{2}:=\Gamma_{R}^{2}\left(z_{2}^{2}, z_{1}^{2}\right), \Gamma_{R}^{3}:=\Gamma_{R}^{3}\left(z_{1}^{2}, z_{1}^{1}\right)$, $\Gamma_{R}^{4}:=\Gamma_{R}^{4}\left(z_{2}^{1}, z_{2}^{2}\right)$ and $\Gamma_{R}:=\bigcup_{j=1}^{4} \Gamma_{R}^{j}$. Furthermore, $\gamma_{i}^{j}(R)$ is a subset of $\gamma_{i}^{j}$ combining \pm 1 with $z_{i}^{j}$; $L_{R}^{j}:=\gamma_{1}^{j}(R) \cup \gamma_{2}^{j}(R) \cup \Gamma_{R}^{j}$ and $U_{j}:=\operatorname{int}\left(L_{R}^{j} \cup \Gamma^{j}\right)$.

The conformal mapping $\varphi_{0}$ is extended to as follows:

$$
\varphi_{0}\left(z ; z_{0}\right):=\left\{\begin{array}{cc}
\varphi_{0}\left(z ; z_{0}\right) & ; z \in \bar{G} \\
\left(\varphi_{0} o \alpha_{j}^{*}\right)\left(z ; z_{0}\right) & ; z \in U_{j}, j=1,2
\end{array}\right.
$$

Applying the Cauchy-Pompeiu's formula [17, p.148] to the conformal mapping $\varphi_{0}(z)$ we obtain for $z \in G$

$$
\varphi_{0}\left(z ; z_{0}\right)=\frac{1}{2 \pi i} \int_{L_{R}^{1} \cup L_{R}^{2}} \frac{\varphi_{0}\left(\zeta ; z_{0}\right)}{\zeta-z} d \zeta-\frac{1}{\pi} \iint_{U_{1} \cup U_{2}} \frac{\varphi_{0, \bar{\zeta}}\left(\zeta ; z_{0}\right)}{\zeta-z} d \sigma_{\zeta} .
$$

Now consider above the notation we get

$$
\begin{aligned}
\varphi_{0}\left(z ; z_{0}\right)= & \frac{1}{2 \pi i} \int_{\Gamma_{R}} \frac{g\left(z ; z_{0}\right)}{\zeta-z} d \zeta+\frac{1}{2 \pi i} \sum_{i, j=1}^{2} \int_{\gamma_{i}^{\prime}(R)} \frac{\tilde{\varphi}_{0}\left(\zeta ; z_{0}\right)-\varphi_{0}\left((-1)^{j}\right)}{\zeta-z} d \zeta- \\
& -\frac{1}{\pi i} \iint_{U_{1} \cup U_{2}} \frac{\tilde{\varphi}_{0, \bar{\zeta}}\left(\zeta ; z_{0}\right)}{\zeta-z} d \sigma_{\zeta},
\end{aligned}
$$

where

$$
g\left(\zeta ; z_{0}\right):=\left\{\begin{array}{cc}
\tilde{\varphi}_{0}\left(\zeta ; z_{0}\right) & ; \zeta \in \Gamma_{R}^{1} \cup \Gamma_{R}^{2} \\
\varphi_{0}\left(1 ; z_{0}\right) & ; \zeta \in \Gamma_{R}^{3} \\
\varphi_{0}\left(-1 ; z_{0}\right) & ; \zeta \in \Gamma_{R}^{4}
\end{array} .\right.
$$

Lemma 4.1. Le $G \in \widetilde{P Q}(K, \alpha, \beta)$ for some $K>1,0<\alpha<1, \beta \geq 0$. Then for any $n \geq 2$, we have

$$
\left\|\varphi^{\prime}\left(z ; z_{0}\right)-\pi_{n}^{\prime}\left(z ; z_{0}\right)\right\|_{L_{2}(G)} \preccurlyeq \delta^{-\frac{1}{2}}(B)\left(\frac{1}{\ln n}\right)^{\frac{1-\alpha}{2 \alpha}}
$$


Lemma 4.2. Let $G \in \widetilde{P Q}(K, 0, \beta)$ for some $K>1, \quad \beta>0$. Then for any $n \geq 2$, small enough $\varepsilon>0$ we have

$$
\left\|\varphi^{\prime}\left(z ; z_{0}\right)-\pi_{n}^{\prime}\left(z ; z_{0}\right)\right\|_{L_{2}(G)} \preccurlyeq \delta^{-\frac{1}{2}}(B)(\ln n)^{\frac{3 \beta}{2}}\left(\frac{1}{n}\right)^{\frac{1-\varepsilon}{2 K^{2}}}
$$

Proof. The proof of the lemmas 4.1 and 4.2 will be made together, because there is no difference in their proofs. Furthermore, we use the standard method as in [5,9]. Since the first expression on the right hand of (4.2) is analytic in $\bar{G}$, there exists a polynomial $p_{n}(z)$ [20, p.142] such that

$$
\left|\frac{1}{2 \pi i} \int_{\Gamma_{R}} \frac{\mathrm{g}\left(\zeta ; \mathrm{z}_{0}\right)}{(\zeta-\mathrm{z})^{2}} \mathrm{~d} \zeta-\mathrm{p}_{\mathrm{n}}^{\prime}\left(\mathrm{z} ; \mathrm{z}_{0}\right)\right| \preccurlyeq \frac{1}{n}, \mathrm{z} \in \overline{\mathrm{G}}
$$

Thus from (4.3), we obtain

$$
\begin{aligned}
& \left\|\varphi_{0}^{\prime}\left(z ; z_{0}\right)-p_{n}^{\prime}\left(z ; z_{0}\right)\right\|_{L_{2}(G)} \preccurlyeq \\
& n^{-1}+\sum_{i, j=1}^{2}\left\|\int_{\gamma_{i}^{\prime}(R)} \frac{\tilde{\varphi}_{0}\left(\zeta ; z_{0}\right)-\varphi_{0}\left((-1)^{j}\right)}{(\zeta-z)^{2}} d \zeta\right\|_{L_{2}(G)}+\left\|\iint_{U_{1} \cup U_{2}} \frac{\tilde{\varphi}_{0, \zeta}\left(\zeta ; z_{0}\right)}{(\zeta-z)^{2}} d \sigma_{\zeta}\right\|_{L_{2}(G)}=: \frac{1}{n}+\sum_{k=1}^{5} J_{k}
\end{aligned}
$$

According to (3.1) and Lemma 3.3, we have for $j=\overline{1,2}$

$$
\begin{gathered}
\left|\tilde{\varphi}_{0}\left(\zeta ; z_{0}\right)-\varphi_{0}(1)\right|=\left|\varphi_{0}\left(\alpha_{j}^{*}(\zeta) ; z_{0}\right)-\varphi_{0}(1)\right| \preccurlyeq \delta^{-\frac{1}{2}}(B)|\zeta-1|^{\frac{1}{2}}, \zeta \in \gamma_{1}^{j}(R) ; \\
\left|\tilde{\varphi}_{0}\left(\zeta ; z_{0}\right)-\varphi_{0}(-1)\right|=\left|\varphi_{0}\left(\alpha_{j}^{*}(\zeta) ; z_{0}\right)-\varphi_{0}(-1)\right| \preccurlyeq \delta^{-\frac{1}{2}}(B)|\zeta+1|^{\frac{1}{2}}(-\ln |\zeta+1|)^{\frac{\beta}{2}}, \zeta \in \gamma_{2}^{j}(R) .
\end{gathered}
$$

Then, we get from Lemma 3.1 and Lemma 3.2, with $l_{j, i}=m e s \gamma_{j}^{i}(R), i, j=1,2$.

$$
\begin{gathered}
\left\|\int_{\gamma_{1}^{j}(R)} \frac{\tilde{\varphi}_{0}\left(\zeta ; z_{0}\right)-\varphi_{0}(1)}{(\zeta-z)^{2}} d \zeta\right\|_{L_{2}(G)} \leqslant \delta^{-\frac{1}{2}}(B) l_{1, i}^{\frac{1-\alpha}{2}}, 0<\alpha<1, \beta \geq 0 \\
\left\|\int_{\gamma_{2}^{\prime}(R)} \frac{\tilde{\varphi}_{0}\left(\zeta ; z_{0}\right)-\varphi_{0}(-1)}{(\zeta-z)^{2}} d \zeta\right\|_{L_{2}(G)} \leqslant \delta^{-\frac{1}{2}}(B)\left|\ln l_{2, i}\right|^{\beta} \ell_{2, i}^{\frac{1}{2}}, \alpha=0, \beta>0
\end{gathered}
$$

and combining (4.4), (4.6) and (4.7), we get

$$
\sum_{k=1}^{4} J_{k} \preccurlyeq \delta^{-\frac{1}{2}}(B) \cdot\left\{\begin{array}{cl}
l_{1, i}^{\frac{1-\alpha}{2}} & ; 0<\alpha<1, \beta=0 \\
l_{1, i}^{\frac{1-\alpha}{2}}+\left|\ln l_{2, i}\right|^{\beta} l_{2, i}^{\frac{1}{2}} & ; 0<\alpha<1, \beta>0 \bigcup_{i=1}^{n} X_{i} \\
\left|\ln l_{2, i}\right|^{\beta} l_{2, i}^{\frac{1}{2}} & ; \alpha=0, \beta>0 .
\end{array}\right.
$$

Moreover, from [7, Lemma 2.1] and (3.2), we get $d\left(z_{2}^{j}, \Gamma^{j}\right) \preccurlyeq n^{-\frac{1-\varepsilon}{K^{2}}}$ for arbitrary small $\varepsilon>0$. Now, we use the qualities of the conformal mappings $w=\Phi(z), w=\varphi_{0}\left(z ; z_{0}\right)$ in a certain neighborhood of the cusp points and from (3.1) and [7, Lemma 2.3], we obtain

$$
l_{j, i} \preccurlyeq\left|z_{j}^{i}-(-1)^{j+1}\right| \preccurlyeq\left\{\begin{array}{cl}
(\operatorname{lnn})^{-\alpha^{-1}} & ; i=1,2, \quad j=1, \quad \alpha>0 \\
d\left(z_{2}, \Gamma^{j}\right)\left(-\operatorname{lnd}\left(z_{2}, \Gamma^{j}\right)\right)^{\beta} \leqslant(\operatorname{lnn})^{\beta} n^{\frac{\varepsilon-1}{K^{2}}} & ; i=1,2, \quad j=2, \quad \beta>0 .
\end{array}\right.
$$


Then with the help of (4.6) and (4.7), we get

$$
\begin{gathered}
\left\|\int_{\gamma_{1}^{j}(R)} \frac{\tilde{\varphi}_{0}\left(\zeta ; z_{0}\right)-\varphi_{0}(1)}{(\zeta-z)^{2}} d \zeta\right\|_{L_{2}(G)} \leqslant \delta^{-\frac{1}{2}}(B)(\ln n)^{\frac{\alpha-1}{2 \alpha}}, 0<\alpha<1, \beta \geq 0 \\
\left\|\int_{\int_{\gamma / 2}^{j}(R)} \frac{\tilde{\varphi}_{0}\left(\zeta ; z_{0}\right)-\varphi_{0}(-1)}{(\zeta-z)^{2}} d \zeta\right\|_{L_{2}(G)} \leqslant \delta^{-\frac{1}{2}}(B)(\ln n)^{\frac{3 \beta}{2}}\left(\frac{1}{n}\right)^{\frac{1-\varepsilon}{2 K^{2}}}, \alpha=0, \beta>0 .
\end{gathered}
$$

Combining (4.10) and (4.11), we obtain

$$
\sum_{k=1}^{4} J_{k} \preccurlyeq \delta^{-\frac{1}{2}}(B) \cdot\left\{\begin{array}{cc}
(\ln n)^{\frac{\alpha-1}{2 \alpha}} & ; 0<\alpha<1 \\
(\ln n)^{\frac{3 \beta}{2}}\left(\frac{1}{n}\right)^{\frac{1-\varepsilon}{2 K^{2}}} & ; \alpha=0, \beta>0 .
\end{array}\right.
$$

It is known that the Hilbert transformation is bounded linear operator and (3.1) yields

$$
J_{5} \preccurlyeq\left(\sum_{j=1}^{2} \operatorname{mes} \varphi_{0}\left(\alpha^{*}\left(U_{j}\right), z_{0}\right)\right)^{\frac{1}{2}}
$$

Let us define the following statements

$$
\begin{gathered}
V_{1}^{j}:=\left\{\zeta \in \alpha_{j}^{*}\left(U_{j}\right):|\zeta-1| \leq c(\ln n)^{-\alpha^{-1}}\right\}, V_{2}^{j}:=\alpha_{j}^{*}\left(U_{j}\right) \backslash V_{1}^{j}, j=1,2, \alpha>0 . \\
U_{\varepsilon}:=\{\zeta:|\zeta+1| \leq \varepsilon\} ; \tilde{V}_{j}^{1}:=U_{j} \cap U_{\varepsilon}, j=1,2, \alpha=0 .
\end{gathered}
$$

Thus, from [2, Lemma 3.4] and (4.13), we get

Therefore, by (4.13)

$$
\begin{gathered}
\operatorname{mes} \varphi_{0}\left(V_{1}^{j}\right) \preccurlyeq \delta^{-1}(B)(\ln n)^{-\alpha^{-1}} ; \operatorname{mes} \varphi_{0}\left(\alpha_{j}^{*}\left(\tilde{V}_{j}^{1}\right), z_{0}\right) \preccurlyeq \delta^{-1}(B)(n)^{\frac{\varepsilon-1}{K^{2}}} \\
\operatorname{mes} \alpha_{j}^{*}\left(U_{j} \backslash V_{j}^{1}\right) \preccurlyeq \delta^{-1}(B)(n)^{\frac{\varepsilon-1}{K^{2}}}
\end{gathered}
$$

$$
J_{5} \preccurlyeq \delta^{-1}(B)\left\{\begin{array}{cc}
(\ln n)^{-\frac{1}{2 \alpha}} & ; \alpha>0 \\
n^{\frac{\varepsilon-1}{2 K^{2}}} & ; \alpha=0
\end{array}\right.
$$

Consequently, from (4.4), (4.12) and (4.14), we obtain for small enough $\varepsilon>0$

$$
\left\|\varphi_{0}^{\prime}-p_{n}^{\prime}\right\|_{L_{2}(G)} \preccurlyeq \delta^{-\frac{1}{2}}(B)\left\{\begin{array}{cc}
(\ln n)^{\frac{\alpha-1}{2 \alpha}} & ; 0<\alpha<1, \beta \geq 0 \\
(\ln n)^{\frac{3 \beta}{2}}(n)^{\frac{\varepsilon-1}{2 K^{2}}} & \alpha=0, \beta>0 .
\end{array}\right.
$$

Now letting $\tilde{p}_{n}\left(z ; z_{0}\right):=p_{n}\left(z ; z_{0}\right)-p_{n}\left(z_{0} ; z_{0}\right)+\left(\varphi_{0}^{\prime}\left(z_{0} ; z_{0}\right)-p_{n}^{\prime}\left(z_{0} ; z_{0}\right)\right)\left(z-z_{0}\right)$, then $\tilde{p}_{n}\left(z_{0} ; z_{0}\right)=0, \tilde{p}_{n}^{\prime}\left(z_{0} ; z_{0}\right)=1$ and according to means value theorem, we get

$$
\left\|\varphi_{0}^{\prime}\left(z ; z_{0}\right)-\tilde{p}_{n}^{\prime}\left(z ; z_{0}\right)\right\|_{L_{2}(G)} \preccurlyeq\left(1+\delta^{-1}\left(z_{0}\right)\right)\left\|\varphi_{0}^{\prime}\left(z ; z_{0}\right)-p_{n}^{\prime}\left(z ; z_{0}\right)\right\|_{L_{2}(G)}
$$

Since $\varphi=r_{0} \varphi_{0}$ where $r_{0}=\left[\varphi_{0}^{\prime}\left(z_{0}, z_{0}\right)\right]^{-1} \approx \delta\left(z_{0}\right)$, we let $s_{n}:=r_{0} \tilde{p}_{n}$. Thus (4.16) yields 


$$
\left\|\varphi^{\prime}\left(z ; z_{0}\right)-s_{n}^{\prime}\left(z ; z_{0}\right)\right\|_{L_{2}(G)} \preccurlyeq \delta^{-\frac{1}{2}}(B)\left\{\begin{array}{cc}
(\ln n)^{\frac{\alpha-1}{2 \alpha}} & ; 0<\alpha<1, \beta \geq 0 \\
(\ln n)^{\frac{3 \beta}{2}}(n)^{\frac{\varepsilon-1}{2 K^{2}}} & \alpha=0, \beta>0 .
\end{array}\right.
$$

Thus, by extremely property of the Bieberbach polynomials $\pi_{n}\left(z, z_{0}\right)$, Lemmas 4.1 and 4.2 are proved.

\section{The Proofs of the Main Theorems 2.1-2.3}

In this part, we implement a known process given in [16] to the proofs of the main results.

Lemma 5.1. [16] let $\eta_{n}(B):=\sup \left\{\left\|\varphi^{\prime}\left(., z_{0}\right)-\pi_{n}^{\prime}\left(., z_{o}\right)\right\|_{L_{2}(G)}^{2}: z_{0} \in B \breve{G} G\right\}$, then

$$
\omega_{n}(B) \preccurlyeq \delta^{-2}\left(z_{0}\right) \eta_{n}(B)
$$

Now, the proofs of Theorems 2.1- 2.3 are got easily follow from Lemma 5.1 and Lemmas 4.14.2 , depending on the statement of the cusps.

\section{References}

[1] Abdullayev F.G. 1986. On the orthogonal polynomials in domains with quasiconformal boundary (Russian). Dissertation, Donetsk.

[2] Abdullayev F.G., Baki A. 2001. On the convergence of Bieberbach polynomials in domains with interior zero angles. Complex Variables Theory Appl., 44 (2): 131-144.

[3] Abdullayev F.G. 1989. On the convergence of Fourier series by orthogonal polynomials in domains with piecewise-quasiconformal boundary. Theory of Mapping and Approx., Kiev, Naukova Dumka, 3-12.

[4] Abdullayev F.G. 1997. Uniform convergence of the generalized Bieberbach polynomials in regions with non-zero angles. Acta Math. Hung., 77 (3): 223-246.

[5] Abdullayev F.G. 2001. Uniform convergence of the generalized Bieberbach polynomials in regions with zero angles. Czechoslovak Math. J., 51 (126): 643-660.

[6] Abdullayev F.G. 2001. Uniform convergence of the Bieberbach polynomials inside and on the closure of domain in the complex plane. East J. Approx., 7 (1): 77-101.

[7] Abdullayev F.G. 2019. Uniform convergence of the generalized Bieberbach polynomials in regions with simultaneously exterior and interior zero angles. Complex Analysis and Operator Theory, 13 (6): 2881-2897.

[8] Ahlfors L.V. 1966. Lectures on Quasiconformal Mappings, Van Nostrand, Princeton.

[9] Andrievskii V.V. 1983. Uniform convergence of Bieberbach polynomials in domains with piecewise-quasiconformal boundary. (Russian), Theory of Mapping and Approx. Functions, Kiev, Naukova Dumka, 3-18.

[10] Andrievskii V.V. 1983. Convergence of Bieberbach polynomials in domains with quasiconformal boundary. Ukr. Math. J., 35: 233-236.

[11] Gaier D. 1988. On the convergence of Bieberbach polynomials in regions with corners. Const. Approx. 4: 289-305.

[12] Gaier D. 1994. Polynomial approximation of conformal maps. Const. Approx. 14: 27-40.

[13] Israfilov D.M., Oktay B., 2006. Approximation properties of the Bieberbach polynomials in closed Dini smooth domains. Bull. Belg. Math. Soc. 13 (2006): 91-99.

[14] Israfilov D.M., 2001. Approximation of p-Faber-Laurent rational functions in the weighted Lebesque space and the Bieberbach polynomials. Const. Approx., 17 (2001): 335-351.

[15] Keldych M.V. 1939. Sur I'approximation en moyenne quadratique des fontions analtiques. Math.Sb., 5(47): 391-401.

[16] Küçükaslan M., Tunç T., Abdullayev F.G. 2006. Convergence of Bieberbach polynomials inside domains of complex plane. Bull. Belg. Math. Soc., 13 (2006): 657-671. 
[17] Letho O., Virtanen K.I. 1973. Quasiconformal mappings in the plane, Springer-Verlag Berlin.

[18] Mergelyan S.N. 1951. Certain questions of the constructive theory of functions (Russian), Trudy Math. Inst. Steklova, 37: 3-51.

[19] Simonenko I.B. 1980. On the convergence of Bieberbach polynomials in the case of Lipschitz domain. Math. USSR-Izv., 13: 166- 174.

[20] Simornov V.I., Lebedev N.A., 1968. Functions of a complex variable. Constructive Theory. M.I.T. press, Cambridge.

[21] Suetin P.K. 1971. Polynomials orthogonal over the region and Bieberbach polynomials. Proc. Steklov Inst. Math, vol: 100 providence RI: American Mathematical Society.

[22] Wu X.M., 1951. On Bieberbach polynomials. Acta Math. Sinica, 13 (1963):145-151. 